\title{
The Role of Bivalirudin in Percutaneous Coronary Intervention
}

\section{Mohamed Effat*and Said Alsidawi}

Division of Cardiovascular Health and Disease, University of Cincinnati, USA

Bivalirudin (angiomax) is a reversible direct thrombin inhibitor. It is a polypeptide that has a carboxy-terminal region that recognizes thrombin's fibrinogen-binding site, and an amino-terminal tetrapeptide that inhibits the active site of thrombin. It is considered superior to its prototype; hirudin as it is less dependent on renal function and has a lower incidence of anaphylaxis. Bivalirudin has a half-life of 25 minutes and is primarily eliminated via proteolytic cleavage. Compared to heparin, direct thrombin inhibitors, such as bivalirudin, exhibit less variable level of anticoagulation, and they inactivate both free as well as fibrin-bound thrombin. [1]

In most cardiac catheterization laboratories across the United States, Bivalirudin is used mainly as a heparin substitute such as in patients intolerant to heparin (e.g. history of HIT syndrome). However, Bivalirudin clinical studies have demonstrated consistent positive outcomes in patients with stable angina, unstable angina (UA), non-ST segment elevation myocardial infarction (NSTEMI), and ST-segment elevation myocardial infarction (STEMI) undergoing percutaneous coronary intervention (PCI). This therapy has also gained class I recommendation from the ACC/AHA updated guidelines for all of the above mentioned conditions. [2,3]

The Bivalirudin Angioplasty Trial (BAT) was a randomized, doubleblinded, multi-center trial that included 4098 patients undergoing PCI for unstable angina. In this trial, the composite endpoint of death, MI or revascularization was lower in the bivalirudin arm at 90 days with absolute benefits being sustained through 180 days. There was also a significant reduction in the rate of bleeding in the bivalirudin arm compared to heparin. [4]

Similarly, the Randomized Evaluation of PCI Linking Angiomax to Reduced Clinical Events (REPLACE)-1 trial evaluated the efficacy of bivalirudin relative to heparin in patients undergoing PCI. Glycoprotein (GP) IIB/IIIA inhibitors were used in $72 \%$ of patient and clopidogrel was used in $56 \%$ of patients. The incidence of composite end point of death, myocardial infarction, or repeat revascularization was no significantly different between the bivalirudin and heparin groups ( $5.6 \%$ and $6.9 \%$ respectively, $\mathrm{p}=0.40$ ). Major bleeding occurred in $2.1 \%$ versus $2.7 \%$ of patients randomized to bivalirudin or heparin, respectively $(\mathrm{p}=0.52)$. [5]

In the Randomized Evaluation of PCI Linking Angiomax to Reduced Clinical Events (REPLACE)-2 trial, patients were assigned to either bivalirudin, with provisional use of GP IIb/IIIa inhibitor or low-dose unfractionated heparin (UFH) plus planned GP IIb/IIIa inhibitor. There was similar rate of 30-day primary composite endpoint of death, MI, urgent revascularization, and in-hospital major bleeding between the bivalirudin and the heparin-GP IIb/IIIa groups. [6] However, bivalirudin led to significantly less in-hospital major bleeding when compared to the heparin-GP IIb/IIIa inhibitor combination $(2.4 \%$ versus $4.1 \%, \mathrm{p}<0.001)$. There was a difference in mortality favoring bivalirudin at 30 days and 6 months, and 12 months.

Higher risk patients with UA/NSTEMI who were considered for an early invasive strategy were evaluated in the Bivalirudin for Patients with Acute Coronary Syndromes-(Acuity Trial). In this pivotal trial, 13,819 patients were assigned to one of three anti-thrombotic regimens: unfractionated heparin or enoxaparin plus a glycoprotein IIb/IIIa inhibitor, bivalirudin plus a glycoprotein IIb/IIIa inhibitor, or bivalirudin alone. The rates of 30 day ischemic outcomes were similar between bivalirudin plus GP IIb/IIIa inhibitor compared with heparin plus GP IIb/IIIa inhibitor (7.7\% and 7.3\%, respectively). There were also similar rates of major bleeding (5.3\% and 5.7\%). As compared with heparin plus GP IIb/IIIa inhibitor, bivalirudin, alone, led to noninferior rates of ischemia $(7.8 \%$ and $7.3 \%$, respectively; $\mathrm{P}=0.32)$, and significantly reduced major bleeding $(3.0 \%$ vs. $5.7 \%$; $\mathrm{P}<0.001)$. [7]

Bivalirudin also underwent evaluation in the setting of STEMI patients. In the Bivalirudin during Primary PCI in Acute Myocardial Infarction(HORIZONS-AMI trial) [8]. This was a multicenter trial that enrolled 3602 patients with ST-segment elevation myocardial infarction who were undergoing primary PCI. The patients were randomized to a regimen of heparin plus a GP IIb/IIIa inhibitor versus bivalirudin alone. Treatment with bivalirudin alone, as compared with heparin plus GP IIb/IIIa inhibitors was associated with a significant reduction in the 30-day primary endpoint of net adverse clinical events which was predominantly driven by a lower rate of major bleeding. It also lowered 30 -day rates of death from cardiac causes $(1.8 \%$ vs. $2.9 \% \mathrm{P}=0.03)$ as well as death from all causes $(2.1 \%$ vs. $3.1 \% \mathrm{P}=0.047)$. Follow up at one, two, and three years showed that the reduction in the incidence of net adverse clinical events and major bleeding was robustly maintained in the bivalirudin monotherapy group. [8]

\section{Conclusion}

Bivalirudin represents an attractive option as an antithrombotic agent in the cardiac catheterization laboratory. It has several advantages over heparin, and based upon evidence from many clinical trials, it has been proven to be safer than and at least as effective as heparin in the STEMI, NSTEMI/UA, and elective PCI population. We expect that the use of this agent as an antithrombotic therapy during PCI will continue to gain traction in the next few years.

\section{Reference}

1. Shammas NW, Bivalirudin (2005) Pharmacology and clinical applications. Cardiovasc Drug Rev. Winter 23: 345-360.

2. Kushner F G, Hand M, Smith Jr S C, King Sb, S B Anderson et al. (2009) 2009 Focused Updates: ACC/AHA Guidelines for the Management of Patients with ST-Elevation Myocardial Infarction (Updating the 2004 Guideline and 2007 Focused Update) and ACC/AHA/SCAI Guidelines on Percutaneous Coronary Intervention (Updating the 2005 Guideline and 2007 Focused Update. J Am Coll Cardiol 54: 2205-2241.

3. Anderson J L Adams C D, Antman E M Bridges C R, Califf R M et al (2007) ACC/AHA 2007 Guidelines for the Management of Patients with Unstable Angina/Non-ST-Elevation Myocardial Infarctio. J Am Coll Cardiol. 50: e1e157.

4. Bittl JA, Chaitman BR, Feit F, Kimball W, Topol EJ et al. (2001) Bivalirudin versus heparin during coronary angioplasty for unstable or post infarction

*Corresponding author: Mohamed Effat, Division of Cardiovascular Health and Disease, University of Cincinnati, USA, Tel: 513-558-4306; E-mail: effatma@ucmail.uc.edu

Received December 04, 2013; Accepted December 07, 2013; Published December 14, 2013

Citation: Effat M, Alsidawi S (2013) The Role of Bivalirudin in Percutaneous Coronary Intervention. Cardiol Pharmacol 2: e120. doi:10.4172/2329-6607.1000e120

Copyright: ( $) 2013$ Effat M, et al. This is an open-access article distributed under the terms of the Creative Commons Attribution License, which permits unrestricted use, distribution, and reproduction in any medium, provided the original author and source are credited. 
Citation: Effat M, Alsidawi S (2013) The Role of Bivalirudin in Percutaneous Coronary Intervention. Cardiol Pharmacol 2: e120. doi:10.4172/2329$6607.1000 \mathrm{e} 120$

Page 2 of 2

angina: Final report reanalysis of the Bivalirudin Angioplasty Study. Am Heart J 142: 952-959.

5. Lincoff AM, Bittl JA, Kleiman NS, Sarembock IJ, Jackman JD (2004) Comparison of bivalirudin versus heparin during percutaneous coronary intervention (the Randomized Evaluation of $\mathrm{PCl}$ Linking Angiomax to Reduced Clinical Events [REPLACE]-1 trial). Am J Cardiol 93: 1092-1096.

6. Lincoff AM, Kleiman NS, Kereiakes DJ, Feit F, Bittl JA (2004) Long-term efficacy of bivalirudin and provisional glycoprotein Ilb/IIla blockade vs heparin and planned glycoprotein Ilb/llla blockade during percutaneous coronary revascularization: REPLACE-2 randomized trial. JAMA 292: 696703.

7. Stone GW, McLaurin BT, Cox DA, Bertrand ME, Lincoff AM,et al. (2006 Bivalirudin for Patients with Acute Coronary Syndromes. N Engl J Med; 355: 2203-2216.

8. Stone GW, Witzenbichler B, Guagliumi G, Peruga JZ, Brodie BR (2008) Bivalirudin during primary $\mathrm{PCl}$ in acute myocardial infarction. $\mathrm{N}$ Engl $\mathrm{J}$ Med 358: 2218-2230. 